\title{
Seismic waves in the asteroid environment
}

\author{
Paul Sánchez ${ }^{1, *}$ and Daniel J. Scheeres ${ }^{2, * *}$ \\ ${ }^{1}$ Colorado Center for Astrodynamics Research, University of Colorado Boulder, 3775 Discovery Dr, Boulder, CO 80303, USA \\ ${ }^{2}$ Ann \& H.J. Smead Department of Aerospace Engineering Sciences, University of Colorado Boulder, 3775 Discovery Dr, Boulder, \\ CO 80303, USA
}

\begin{abstract}
Through numerical simulations, we investigate impact generated seismic wave transmission in granular media under extremely low pressure. This mimics the conditions in the interior of asteroids and other small planetary bodies. We find a dependency not only on the overburden pressure on the medium, but also on the velocity of the impact that generates the wave. This is, at extremely low values of overburden pressure, the wave speed depends no only on the imposed pressure, but also on the increment in pressure created by the passing of the wave. We study crystalline and random packings and find very similar behaviour though with different wave speeds as expected. We then relate our results to different mission-related events on asteroids.
\end{abstract}

\section{Introduction}

Experimentally, it is known that seismic waves speed in a granular medium is related to the overburden pressure $(P)$ applied to it $[1,2]$. The theory that has been developed is called Effective Medium Theory (EMT) and is based on considering the media as elastic, thus removing the difficulties presented by particle size and shape. This theory, based on the Hertzian laws for contacts, predicts a sound velocity that has a $P^{\frac{1}{6}}$ dependency, whereas the experiments have found this is true only for high enough pressures and that at low pressures the dependency changes to $P^{\frac{1}{4}}$. In fact, given that the sound speed for $\mathrm{P}$ and $\mathrm{S}$ waves are related to the elastic constants of the material, in the long wave limit, these can be written as:

$$
\begin{gathered}
v_{p}=\sqrt{\frac{K+4 / 3 \mu}{\rho},} \\
v_{s}=\sqrt{\frac{\mu}{\rho}},
\end{gathered}
$$

where $K$ is the bulk modulus, $\mu$ is the shear modulus and $\rho$ is the density of the system. However, as far as we know, experiments have been limited to $\mathrm{kPa}$ and $\mathrm{MPa}$ pressures as they have been carried out under Earth's gravity.

Small planetary bodies (asteroids, comets and small moons) produce gravitational fields in the milli- and micro$g$ regimes and, as a consequence, their interior pressure varies from zero (on their surface) to just a few Pascals or tens of Pascals in their innermost regions.

In order to investigate seismic wave transmission in the asteroid environment, we carry out granular dynamics simulations that mimic these low-pressure conditions. We use a soft-sphere discrete element method (SSDEM) code and relate our findings to different space missions.

\section{Method}

The simulation program that is used for this research applies a Soft-Sphere Discrete Element Method (SSDEM) $[3,4]$, implemented as a computational code (in house developed) to simulate a granular aggregate [5-7]. The particles, modelled as spheres that follow a predetermined size distribution, interact through a soft-repulsive potential when in contact. This method considers that two particles are in contact when they overlap. When this happens, normal and tangential contact forces are calculated [8]. The former is modelled by a hertzian spring-dashpot system and is always repulsive, keeping the particles apart; the latter is modelled with a linear spring that satisfies the local Coulomb yield criterion. The normal elastic force is modelled as

$$
\overrightarrow{\mathbf{f}}_{e}=k_{n} \xi^{3 / 2} \hat{\mathbf{n}},
$$

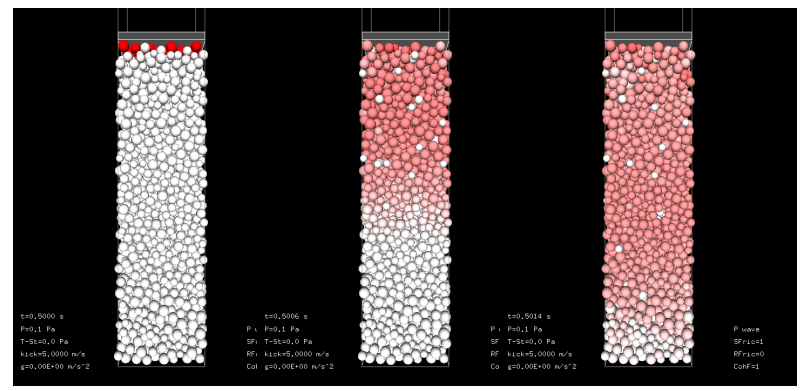

Figure 1. Simulation setup of a randomly packed aggregate; the redder the particle, the greater its vertical speed component $(P=0.1 \mathrm{~Pa})$.

\footnotetext{
*e-mail: diego.sanchez-lana@colorado.edu
}

**e-mail: daniel.scheeres@ colorado.edu

A video is available at https://doi.org/10.48448/t6r1-mn73 
the damping force as:

$$
\overrightarrow{\mathbf{f}}_{d}=-\gamma_{n} \dot{\xi} \hat{\mathbf{n}} .
$$

Then, the total normal force is calculated as $\overrightarrow{\mathbf{f}}_{n}=\overrightarrow{\mathbf{f}}_{e}+\overrightarrow{\mathbf{f}}_{d}$. In these equations, $k_{n}$ is the elastic constant, $\xi$ is the overlap of the particles, $\gamma_{n}$ is the damping constant (related to the dashpot), $\dot{\xi}$ is the rate of deformation and $\hat{\mathbf{n}}$ is the vector joining the centres of the colliding particles. This dashpot models the energy dissipation that occurs during a real collision.

The tangential component of the contact force models static and dynamic surface friction. This is calculated by placing a linear spring attached to both particles at the contact point at the beginning of the collision $[8,9]$ and by producing a restoring frictional force $\overrightarrow{\mathbf{f}}_{t}$. The magnitude of the elongation of this tangential spring is truncated in order to satisfy the local Coulomb yield criterion $\left|\overrightarrow{\mathbf{f}}_{t}\right| \leq \mu\left|\overrightarrow{\mathbf{f}}_{n}\right|$, with $\mu=0.5$.

\section{Procedure}

Here, we have chosen to use material parameters close to those of basalt so that the results are relevant for asteroids; the grains are spherical so that the results can also be related to sound transmission theory (previous sections). We use 3120 grains with diameters between $2-3 \mathrm{~cm}$ that follow a uniform, random distribution for the randomly packed grains (RCP) (see Fig. 1) and 3570, $2.5 \mathrm{~cm}$ grains for the crystalline packing (HCP). Their density is $3200 \mathrm{~kg} \mathrm{~m}^{-3}$, Young modulus is $7.8 \times 10^{10} \mathrm{~N} \mathrm{~m}^{-2}$, Poisson ratio is 0.25 [10]. Two coefficients of restitution are used: 0.1 while the particles are settling and 0.5 for the actual simulations. This reduces the settling time.

The change in particle number was required so that the height of the columns remained the same for all simulations. The different particle arrangements implied different filling fractions and so, different bulk densities. However, we chose to keep the particle density the same and let the bulk density change as this more closely represents realistic laboratory conditions.

The particles are contained in a box with a solid bottom, horizontal periodic boundary conditions and a moving top that allows us to impose a very well determined pressure on the system. Though the particles initially settle under Earth's gravity, this is removed at a later stage to avoid pressure gradients. The height of the settled system is approximately $82 \mathrm{~cm}$ (see Fig. 1).

To initiate the seismic wave, the particles at a distance of at most 1.5 times their radius from the moving top are given a vertical velocity (kick) of $0.01,0.1,0.5$ and $5 \mathrm{~m} / \mathrm{s}$ after $0.5 \mathrm{~s}$ from the moment the settling procedure is finished. The system is then divided into horizontal slices 5 $\mathrm{cm}$ thick to monitor energy transmission. The kinetic energy of each slice is calculated in order to observe the wave transmission. Data is collected every $5 \times 10^{-5} \mathrm{~s}$ after the wave is started and this is done for $0.015 \mathrm{~s}$. Sound speed is measured for the peak of the wave when it reaches the $2^{\text {nd }}$ slice from the bottom. The overburden pressure was varied between 0.1 and $50000 \mathrm{~Pa}$, whereas particle-particle tensile strength is varied between 0 and $1000 \mathrm{~Pa}$.

\section{Results}

As expected from the previous experimental results, pressure has a very noticeable effect on wave speed. We changed the overburden pressure over 5 orders of magnitude and the results were always consistent; as pressure decreases so does the wave speed. The effect of cohesion is not completely clear and so we will focus here on the cohesionless case (see fig.2). Note that wave speeds in the hundreds of $\mathrm{m} / \mathrm{s}$ are in complete agreement with what has been found on Earth (see [11] figs. 1 and 2) for overburden pressures in the tens of $\mathrm{kPa}$. As the pressure diminished, we expected to see a steady decline with wave speeds going down to just a few tens of meters per second for all the tested pressures. Regardless of the specific exponent for the dependency on pressure, theory and experiments have shown that wave speed as $P$ tends to zero, $v_{p} \rightarrow \sqrt{K / \rho}$ which should be constant regardless of the impact velocity; however, this was not what we found.

A question that is obvious to ask is the reason for the formation of the plateau and why the different values for the plateau. The reason for this appears to be the increase in local pressure that the passing wave produces in the medium. In order to understand this, we note that a $\mathrm{P}$ wave is simply a pressure wave and so it should not be a surprise that as the wave passes through the medium, the local pressure changes. If our intuition were true, impacts at different speeds should cause different pressure variations that would be related to a variation in wave speed.

In order to measure the effect of the passing wave on the granular medium, we calculate the stress tensor and
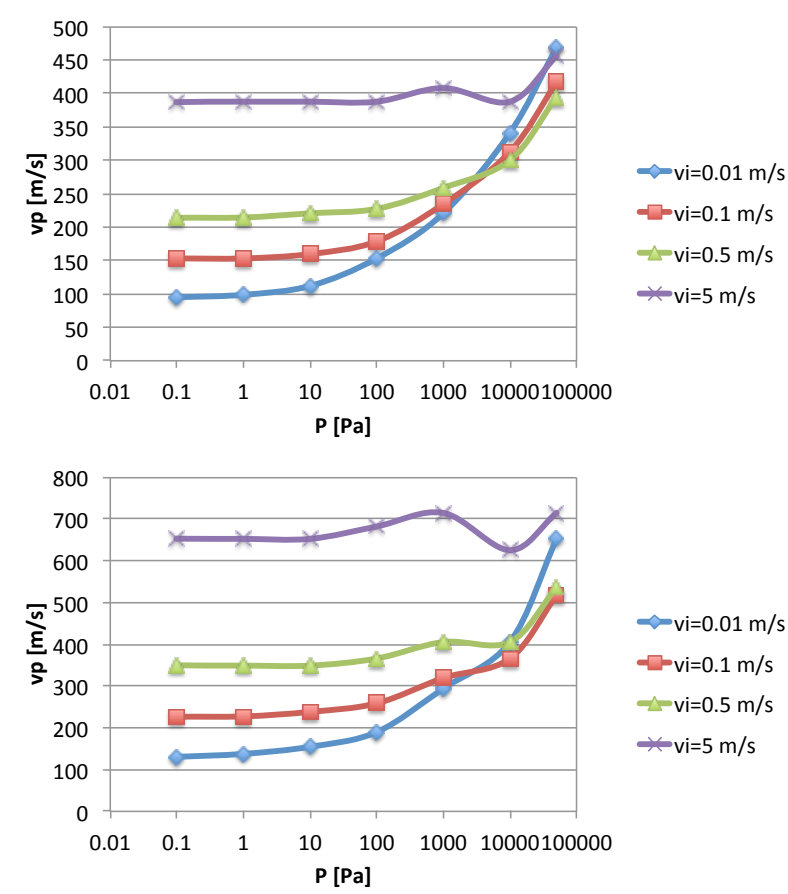

Figure 2. Wave speed versus overburden pressure at different impact speeds (logarithmic scale) for cohesionless grains. Top: RCP, bottom: HCP. 
the principal stresses in each slice. As in our previous research [7], we calculate the average Cauchy stress tensor. This is, the stress tensor or an aggregate of particles contained within a volume $V$ is equal to the sum of the dyadic product of the reaction force $(\vec{f})$ between any two particles in contact and the arm vector $(\vec{l})$ between their centroids [12]. Mathematically:

$$
\bar{\sigma}=\frac{w}{V} \sum_{c \in K_{V}} \vec{f} \otimes \vec{l}
$$

In the above equation $K_{V}$ is the set of contacts between the particles in volume $V$ and the weight function $w$ has a value of 1 for contacts of particles in the same volume and 0.5 when they are in different volumes [13]. Once the stress tensor is calculated, we calculate its eigenvalues and from them, the hydrostatic pressure. For this specific simulation, we will only calculate the stress tensor over the entire container and so $w=1$ always.

Fig. 3 shows the pressure of the entire container vs. time for an impact at $0.5 \mathrm{~m} / \mathrm{s}$ from the moment the wave is started until the arrival of the peak to the point of measure. For these simulations, the particles are completely cohesionless and we have varied only the overburden pressure as indicated in the figure. As it can be clearly observed, though the pressure of all of them is different as it was imposed by the moving top, once the wave was initiated, the total pressure was incremented by $\approx 2100 \mathrm{~Pa}$. As such, a difference in the wave speed was observed only when the sum of the imposed overburden pressure plus the pressure of the wave itself was sufficiently different from the former. For this particular impact velocity, that happened for an overburden pressure of $1000 \mathrm{~Pa}$. The yellow dots in the figure mark the instant when the wave arrived; it can be seen that they cluster at about the same point for simulations with overburden pressures of up to $100 \mathrm{~Pa}$, but as soon as the total pressure is different (as predicted by the theory), the wave arrives sooner, implying an increase in speed. The dynamics of all the other cases are similar and have the same underlying Physics, but there are of course different numerical values.

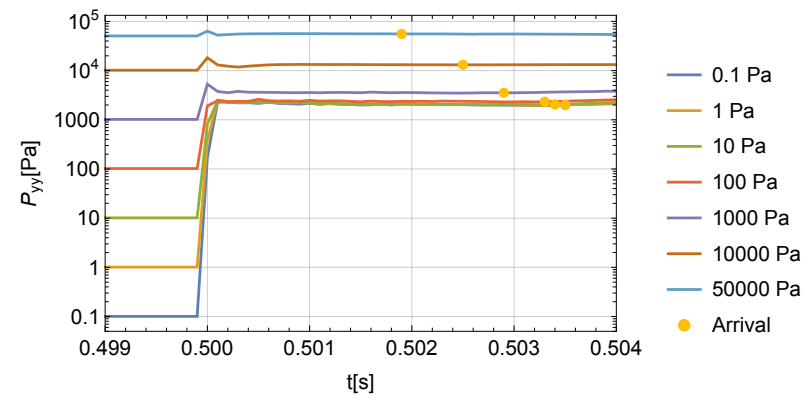

Figure 3. $P_{y y}$ of the particles in the container versus time. Data points were taken $0.005 \mathrm{~s}$ before the initiation of the wave. Different line colour represents a different overburden pressure. The yellow dots show the instant the peak of the wave reached the second slice of particles from the bottom. Cohesionless grains, impact at $0.5 \mathrm{~m} / \mathrm{s}$.
This non-linear behaviour at very low confining pressure has been predicted by the theory [14] and found experimentally [15]. However, the results have not been applied to the evolution of small asteroids in the Solar System or to space exploration missions.

For random packings, the impacts in our simulations have produced an increase in the total pressure of $\approx 10.7$ $\mathrm{Pa}, 206.4 \mathrm{~Pa}, 1992.0 \mathrm{~Pa}$ and 40235.5 $\mathrm{Pa}$ at the time of arrival of the waves for impacts at $0.01,0.1,0.5$ and $5 \mathrm{~m} / \mathrm{s}$ respectively. For HCP simulations, at the moment of arrival, the pressure in the system is approximately $25.7 \mathrm{~Pa}$, $525.3 \mathrm{~Pa}, 4.4 \mathrm{kPa}, 91.5 \mathrm{kPa}$ for impact speeds of $0.01,0.1$, 0.5 and $5 \mathrm{~m} / \mathrm{s}$ respectively. If we compare these numbers with the onset of the plateaus, we can observe an agreement with the pressure at which the plateaus start to be apparent. What this means is that a plateau in wave speed is going to be observed when the overburden pressure is at least comparable to the pressure provided by the passing wave. The wave speed is still related to the pressure of the medium it is just that now the pressure is imposed by the sum of wave-induced pressure and the pressure exerted by our moving top. This can be readily observed in fig. 4 where we have plotted the value of the measured speed for $\mathrm{P}$ waves and internal pressure; the best agreement is for a linear dependence of $v_{p}^{2}$ on $P^{1 / 2}$; however, the data is too noisy to affirm this unequivocally. This result suggests that the found experimental dependence would continue even below the experimentally tested range. At the experimental pressures that are usual in Earthbound settings, these small variations would not be observed. This data would imply that $K$, the bulk modulus can be obtained as
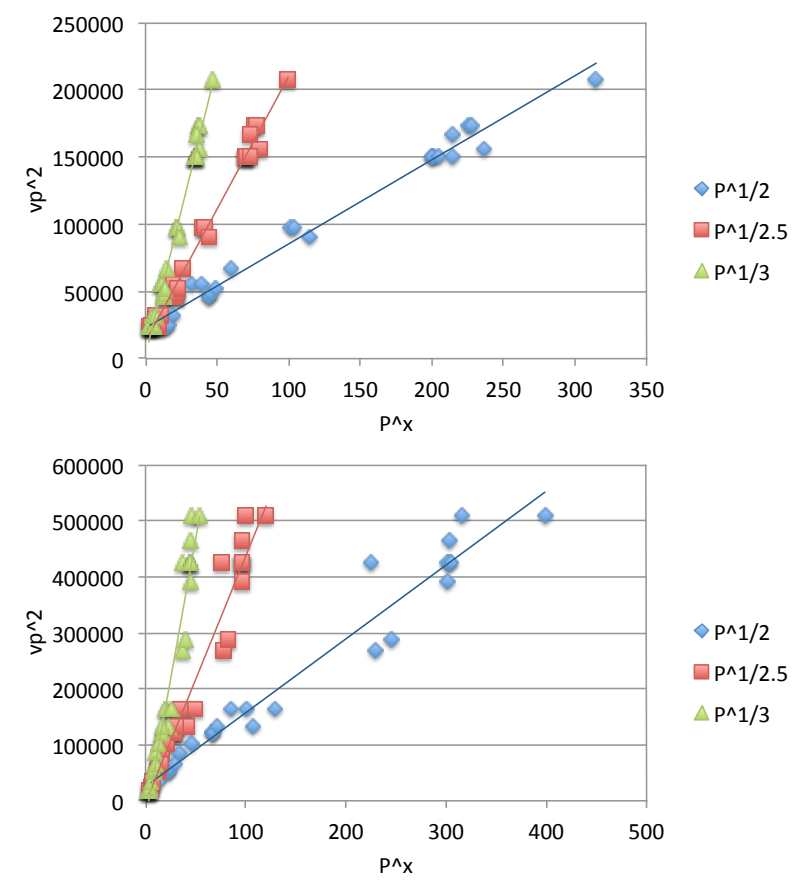

Figure 4. Squared speed of the $\mathrm{P}$ wave vs internal pressure elevated to the powers of $1 / 2,1 / 2.5$ and $1 / 3$ (cohesionless simulations). RCP (top) and HCP (bottom) simulations. 
the limit of Eq. 1 when the internal pressure tends to zero. The lower bound for sound speed of the $\mathrm{P}$ waves in the specific granular medium simulated here would be $\approx 150$ $\mathrm{m} / \mathrm{s}$ and $\approx 159 \mathrm{~m} / \mathrm{s}$ respectively for RCP and HCP structures. At the same time, $K$ would be $\approx 4.7 \times 10^{7} \mathrm{~Pa}$ and $\approx 6 \times 10^{7} \mathrm{~Pa}$ for RCP and HCP structures respectively.

A secondary observation is that by the time the peak of the wave has reached the bottom of the box, most of the energy of the wave had already been dissipated. In fact, dissipation rates start at around $60 \%$ and rapidly go up to about $90 \%$ ( $97 \%$ for HCP systems) as the impact velocity increases. If we take some round numbers for the sake of comparison, let us say that only $10 \%$ of the initial energy can be transmitted after the wave has travelled $1 \mathrm{~m}$ linearly. Which would mean that the average speed of the particles in the wavefront is about just $1 \%$ of the initial velocity of the impactor. Energy transmission then would go as $0.1^{R}$, where $R$ is the distance from the impact point. If we add to this the 3D nature of the system, energy per unit surface area would go as $R^{-2}$. So in general, energy transmission would go roughly as $0.1^{R} R^{-2}$.

If we take this result and apply it to the impact of the Hayabusa 2 mission on asteroid Ryugu, at the $5 \mathrm{~m}$ mark, energy per surface area would have been reduced by a factor of $10^{-7}$ and at the $10 \mathrm{~m}$ mark, the reduction factor would be in the order of $10^{-12}$. The speed of small rocks about the same mass of the SCI would have been reduced by factors of $10^{-3.5}$ and $10^{-6}$ respectively. This is, at the 5 $\mathrm{m}$ mark, we would expect small rocks moving at $0.6 \mathrm{~m} / \mathrm{s}$ and at $0.002 \mathrm{~m} / \mathrm{s}$ at the $10 \mathrm{~m}$ mark as the wave passed. We have not accounted for the energy spent in compressing the very porous surface or the formation of an ejecta field.

\section{Implications for asteroids}

Taking what we have observed in our DEM simulations, and if we accept them as a good enough representation of such a granular system, with the usual caveats about particle shape, size distribution and contact dynamics, there are some things that we could say about impacts and seismic wave transmission on small planetary bodies and possible observations made by the mentioned missions:

- If seismic shaking due to small meteorite impacts on small bodies is not produced, or at least not to the extent that has previously been portrayed, the BNE might not be a possible explanation for global particle size segregation. However, the surface could be segregated [16, 17].

- The planned impact on asteroid Dimorphos (DART mission) should indeed create a large crater and a lot of debris, but the seismic wave might not be able to reach the other side of the body.

- The landing of asteroid Itokawa must have hardly disturbed particles far from the landing sites. At a distance of about $1 \mathrm{~m}$ from this place, most of the energy should have been dissipated.
- The impact and the explosion produced on asteroid Ryugu should not have had much of an effect on the terrain in a radius greater than $5-10 \mathrm{~m}$ for the same reason.

-The sampling manoeuvre planned by the OSIRIS-REx mission, at a nominal speed of $10 \mathrm{~cm} / \mathrm{s}$, should not cause any disturbance in the terrain around it. Note, that this refers only to the impact of the TAGSAM (Touch-and-Go Sample Acquisition Mechanism) head on the surface of Bennu and not to the gas ejection.

- The debris that falls on the surface of Didymos, with impact velocities not greater than $10 \mathrm{~m} / \mathrm{s}$ [18], will not cause large disturbances either though they will cause some secondary ejecta.

\section{References}

[1] K. Walton, Geophys. J. Int. 48, 461 (1977)

[2] P.J. Digby, J. Appl. Mech 48, 803 (1981)

[3] P. Cundall, A Computer Model for Simulating Progressive Large Scale Movements in Blocky Rock Systems, in Proceedings of the International Symposium on Rock Mechanics 1, 129-136 (Nancy, 1971)

[4] P.A. Cundall, R.D. Hart, Eng. Computations 9, 101 (1992)

[5] P. Biswas, P. Sánchez, M. Swift, P. King, Phys. Rev. E 68, 050301(R) (2003)

[6] P. Sánchez, D.J. Scheeres, Astrophys. J. 727, 120 (2011)

[7] D.P. Sánchez, D.J. Scheeres, Icarus 218, 876 (2012)

[8] H. Herrmann, S. Luding, Continuum Mech.Therm. 10, 189 (1998)

[9] L.E. Silbert, D. Ertaş, G.S. Grest, T.C. Halsey, D. Levine, S.J. Plimpton, Phys. Rev. E 64, 051302 (2001)

[10] B. Gundlach, G. Blum, Icarus 223, 479 (2013)

[11] J.D. Goddard, J.E. Enderby, Proceedings of the Royal Society of London. Series A: Mathematical and Physical Sciences 430, 105 (1990)

[12] S.J. Anthony, W. Hoyle, Y. Ding, Granular Materials: Fundamentals and Applications (The Royal Society of Chemistry, Cambridge, UK, 2004)

[13] S. Luding, Particuology 6, 501 (2008)

[14] K. Tell, C. Dreißigacker, A.C. Tchapnda, P. Yu, M. Sperl, Review of Scientific Instruments 91, 033906 (2020)

[15] S. van den Wildenberg, R. van Loo, M. van Hecke, Phys. Rev. Lett. 111, 218003 (2013)

[16] V. Perera, A.P. Jackson, E. Asphaug, R.L. Ballouz, Icarus 278, 194 (2016)

[17] E. Wright, A.C. Quillen, J. South, R.C. Nelson, P. Sánchez, L. Martini, S.R. Schwartz, M. Nakajima, E. Asphaug, Icarus 337, 113424 (2020)

[18] Y. Yu, P. Michel, Icarus 312, 128 (2018) 